\title{
Calcium pyrophosphate dihydrate crystal deposition disease: a case of cervical myelopathy in an elderly woman
}

Cervical cord compression in elderly patients is usually degenerative in origin. We report a case of cervical cord compression due to focal deposition of calcium pyrophosphate dihydrate (CPPD) crystals in the ligamentum flavum.

A 76 year old woman presented with a 3 week history of severe neck pain associated with intermittent L'Hermitt's type electric shock sensations down both arms. Over this period she noticed clumsiness of her hands and became unsteady on her feet. For several years she had complained of mild neck pain and stiffness. She walked with a broad based ataxic gait and had brisk reflexes and hypertonia in both lower limbs with reduced muscle power MRC grade $4 / 5$ below the C7 level bilaterally.

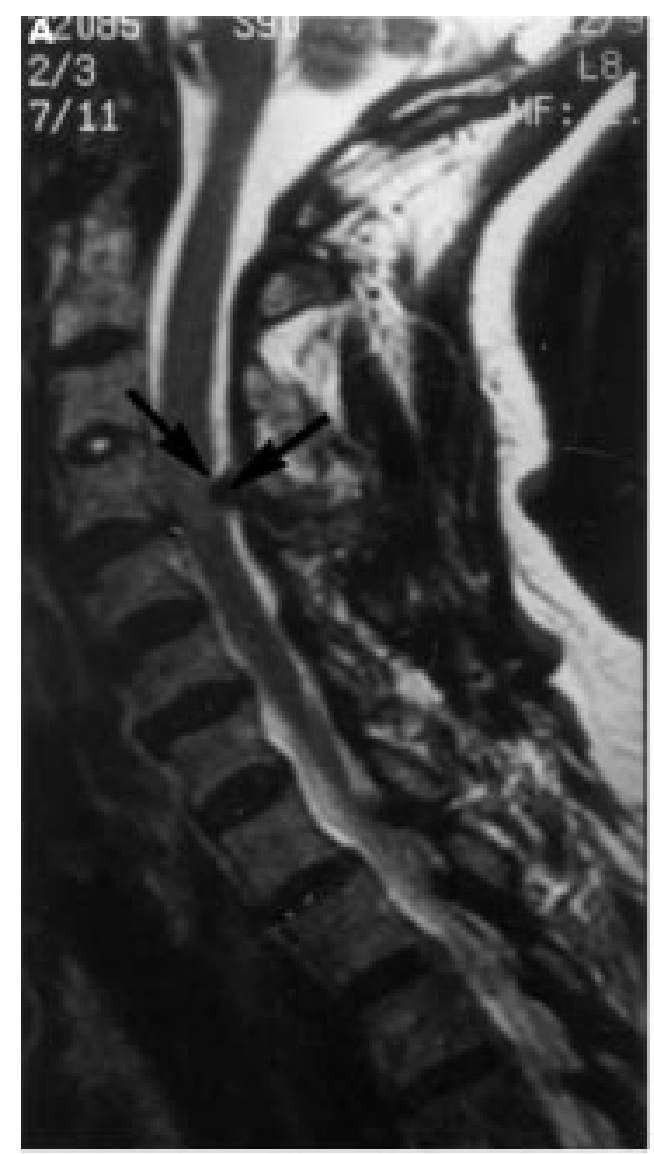

Sagittal (A) and axial (B) MRI T2 weighted images showing the focal lesion (arrows) compressing the spinal cord. (C) Light photomicrograph of CPPD crystals in excised ligamentum flavum.
In the upper limbs, the triceps reflexes were brisk. An MR scan of the cervical spine showed a focal, non-enhancing, posteriorly based soft tissue mass at the $4 / 5$ level extending laterally within the spinal canal in a symmetric fashion and causing cord compression (figure, A, B). The patient underwent laminectomy at $\mathrm{C} 4$ and C5 with excision of a thickened band of ligamentous tissue which was of gritty consistancy and yellow grey in colour. The dura was intact and cord pulsation was noted after decompression. Postoperative recovery was uneventful and the patient was discharged 9 days after surgery.

Histological examination of the excised ligamentum flavum showed fibrocartilaginous
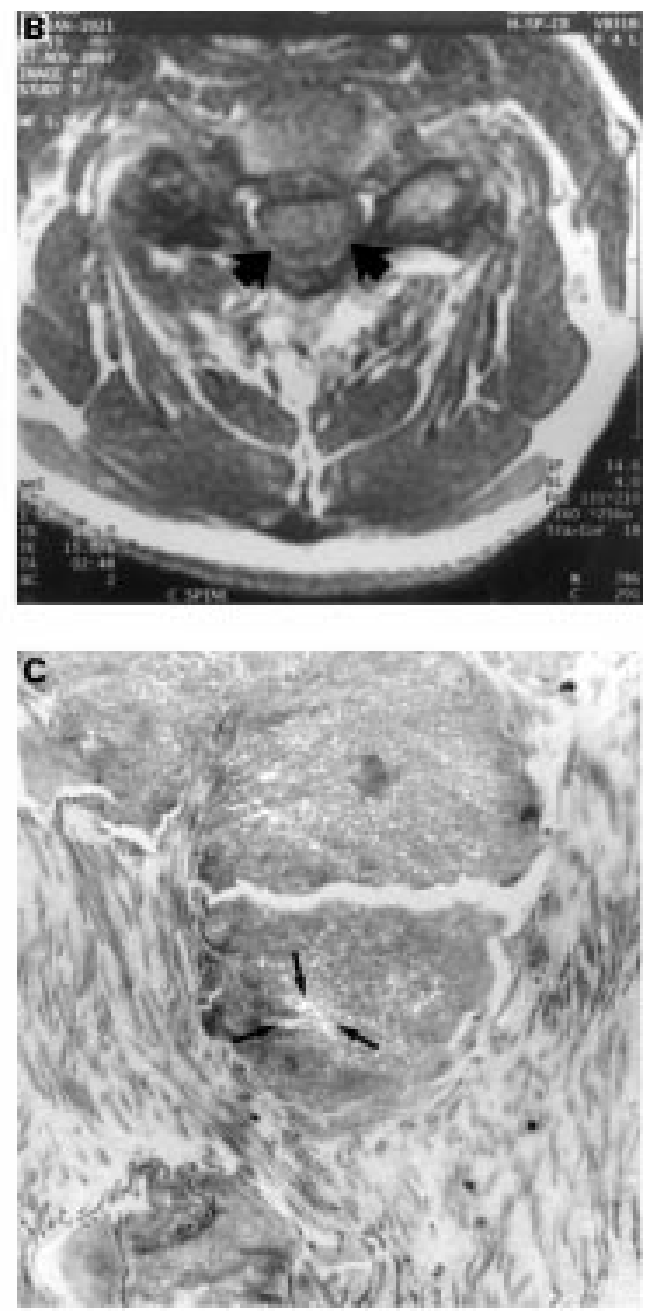
material with deposits of amorphous material containing birefringent crystals when examined under polarised light (figure, C). A diagnosis of CPPD crystal deposition disease was made.

This disease presents as a monoarthropathy or oligoarthropathy most commonly affecting knees, wrists, and metacarpophalangeal joints. The diagnosis is generally based on radiological findings and the detection of crystals on synovial fluid analysis. ${ }^{12}$ Plain films of affected joints show areas of calcification in articular cartilage (chondrocalcinosis). Crystals of CPPD are square or rhomboid in shape and weakly birefringent under polarised light. ${ }^{3}$ There are no specific serological changes associated with CPPD crystal deposition disease but several treatable metabolic and endocrine states can coexist with the condition-for example, hyperparathyroidism, hypothyroidism, haemochromatosis, hypomagnesaemia, and hypophoshphatasia. ${ }^{1}$ We found 10 reports in the literature of patients with CPPD crystal deposition disease who presented with cervical myelopathy. Kawano et al examined the excised fragments of ligamentum flavum in three patients with cervical myelopathy and found, using $x$ ray diffraction studies, that the crystals consist of an outer coat of CPPD and a centre of hydroxyapatite. Electron microscopy showed that CPPD crystals are preferentially deposited in areas of degenerate elastic fibres and they concluded that the cervical ligamentum flavum is favoured for crystal deposition due to its mobility and risk of minor injury. ${ }^{3}$ CPPD crystal deposition disease is overlooked as a differential diagnosis in elderly patients presenting with myelopathy or radiculopathy but has been reported to cause lesions ranging the craniocervical junction to the lumbar spine. ${ }^{4}$ Magnetic resonance imaging is the investigation of choice. This allows the exclusion of prolapsed intervertebral disc and has the advantage of multiplanal visualisation. Deposition of CPPD crystals in the ligamentum flavum can appear as a focal or diffuse mass lesion on MRI. ${ }^{5}$ CPPD causing focal cervical cord compression without arthropathy elsewhere in the body should be managed by decompressive surgery alone. The growing number of reported cases of CPPD crystal deposition disease in the ligamentum flavum serves to justify the inclusion of this condition in the differential diagnosis of myelopathy in the elderly patient.

J CAIRD
G ROBERTS
S YOUNG
Department of Neurosurgery
F BRETT
Department of Neuropathology, Beaumont Hospital,
Dublin 9, Ireland

Correspondence to: Dr J Caird, Department of Neurosurgery, Beaumont Hospital, Dublin 9, Ireland. Telephone 003531 8377755; fax: 0035318092302.

1 Michet CJ Jr, Evans JM, Fleming KC, et al. Common rheumatological diseases in elderly patients. Mayo Clin Proc 1995;70:1205-14

2 McCarty DJ. Calcium pyrophosphate dihydrate crystal deposition disease: nomenclature and diagnostic criteria. Ann Int Med 1977;87:240-2

3 Kawano N, Matsuno T, Miyazawa S, et al. Calcium pyrophosphate dihydrate deposition in the cervical ligamentum flavum. I Neurosurg 1988;68:613-20.

4 Salcman M, Khan A, Symonds DA. Calcium pyrophosphate arthropathy of the spine: case report and review of the literature. Neurosurgery 1994;34:915-18.

5 Brown TR, Quinn SF, D'Agostino AN. Deposition of calcium pyrophosphate dihydrate crystals in the ligamen-
tum flavum: evaluation with MR Imaging and CT. Radioltum flavum: evaluation
ogy 1991;78:871-3. 